Mots. Les langages du politique

Les élus socialistes face aux chiffres de la délinquance. Dispositions, positions et prises de position partisanes sur les statistiques policières

Rafaël Cos

\title{
CpenEdition
}

Journals

Édition électronique

URL : https://journals.openedition.org/mots/20984

DOI : $10.4000 /$ mots. 20984

ISSN : 1960-6001

Éditeur

ENS Éditions

Édition imprimée

Date de publication : 15 décembre 2012

Pagination : 107-122

ISBN : 978-2-84788-387-9

ISSN : 0243-6450

Référence électronique

Rafaël Cos, «Les élus socialistes face aux chiffres de la délinquance. Dispositions, positions et prises de position partisanes sur les statistiques policières », Mots. Les langages du politique [En ligne], 100 | 2012, mis en ligne le 15 décembre 2014, consulté le 23 avril 2022. URL : http://

journals.openedition.org/mots/20984; DOI : https://doi.org/10.4000/mots.20984 


\section{Les élus socialistes face aux chiffres de la délinquance. Dispositions, positions et prises de position partisanes sur les statistiques policières}

Plusieurs travaux récents convergent autour du constat que l'une des transformations majeures de l'action publique tient dans l'usage croissant d'indicateurs chiffrés censés optimiser la conduite de cette dernière (Belorgey, 2010, Bezès, 2009, Bruno, 2008). Le cas des politiques publiques de sécurité ne semble pas échapper à ce mouvement. De nombreuses recherches ont ainsi analysé la mise en place et les effets, depuis les dix dernières années, d'une «culture du résultat» supposée permettre d'améliorer l'efficacité de la police (Matelly, Mouhanna, 2007, Monjardet, 2006, Mucchielli, 2008). L'une des particularités des indicateurs mobilisés à cet effet réside dans la double instrumentalisation, interne et externe, dont ils font l'objet. Censées orienter l'action des services de police et de gendarmerie, les statistiques policières (police et gendarmerie) constituent également - de façon plus récente - un puissant levier de la communication ministérielle et gouvernementale.

Néanmoins, sur les questions de sécurité comme sur d'autres, les travaux traitant des relations entre statistique et politique se bornent au constat que les professionnels de la politique s'en tiendraient à un usage cynique des chiffres dans le cadre de la compétition électorale, sans que les modalités discursives et les ressorts sociaux de cette appropriation ne soient documentés ${ }^{1}$. Cet usage opportuniste des statistiques est de surcroît systématiquement postulé, au risque de réduire l'analyse des usages des chiffres à la seule dimension stratégiste de l'action politique. Ainsi, ni les formes, ni les ressorts de l'inscription proprement politique de ce «langage conventionnel de référence» (Desrosières, 1992) ne sont analysés pour eux-mêmes.

1. La place croissante des chiffres dans le cadre des discours politiques n'a pas encore fait l'objet d'analyses d'ensemble. Pour plusieurs aspects documentés de ce mouvement, se reporter aux travaux récents de Benjamin Lemoine (2008a, 2008b).

Université Lille Nord de France, Centre d'études et de recherches administratives, politiques et sociales rafael.cos@univ-lille2.fr 
Ce travail cherche donc à reconstituer la trame rhétorique et cognitive mettant aux prises le Parti socialiste, alors qu'il occupait le rôle de principal opposant sous la mandature de Nicolas Sarkozy, avec un ensemble de références chiffrées qui ont la particularité d'être autant dominantes dans le débat public qu'elles sont critiquées dans leurs fondations. Que savait l'opposition socialiste de ces chiffres? Comment et pourquoi s'en est-elle servie? Dans quelle mesure les responsables socialistes ont-ils cherché à faire de ces indicateurs d'origine ministérielle un étalon des «échecs » de leurs adversaires? Cet ensemble de questionnements a le double intérêt de mettre en lumière l'économie des controverses interpartisanes en matière de sécurité, en même temps qu'il interroge, de façon plus générale, les conditions du travail politique. L'objectif de cet article est de montrer comment l'usage politique des chiffres renvoie directement aux dispositions, pratiques et représentations des élites partisanes investies dans les enjeux de politiques publiques. La matérialité du travail politique est ici interrogée au prisme des rapports aux savoirs - savoirs d’État ou savoirs académiques - développés par les hommes politiques.

\section{Les statistiques policières au centre de la compétition sur l'enjeu "sécurité "}

Bien saisir les enjeux inhérents au débat sur l'évolution de la délinquance suppose au préalable d'exposer les composantes de la configuration dans laquelle s'insèrent les controverses. Cette configuration intègre des éléments de diagnostic administratifs, des controverses scientifiques et médiatiques sur la pertinence de ces indicateurs, l'action publique engagée par le ministère de l'Intérieur et la stratégie politique du principal parti d'opposition d'alors, le Parti socialiste.

\section{Managérialisation et médiatisation de la politique policière}

L'instrumentalisation politique des chiffres de la délinquance n'est pas nouvelle. Elle remonte à la publicisation de ces chiffres à partir de 1972 et la création de l'État 4001, index statistique chargé de mesurer l'évolution des différents types de crimes et délits à travers le temps². Longtemps mobilisés comme

2. À partir de 1972, ces chiffres font d'abord l'objet d'une diffusion annuelle. Depuis 2002 et l'arrivée de Nicolas Sarkozy au ministère de l'Intérieur, ils sont publiés mensuellement. Jusqu'en 2004, c'est la Direction centrale de la police judiciaire (DCPJ) qui centralise ces chiffres d'abord collectés au niveau des Directions départementales de sécurité publique (DDSP). Depuis cette date, ils font l'objet d'un retraitement statistique de la part de l'Observatoire national de la délinquance (OND), transformé en octobre 2009 en Observatoire national de la délinquance et des réponses pénales (ONDRP). L’Observatoire produit des notes et des rapports réguliers; chaque début d'année civile, un représentant vient livrer une analyse des chiffres en compagnie du ministre à l'occasion d'une conférence de presse prévue à cet effet. Dans la suite de la démonstration, nous employons l'appellation ONDRP pour désigner indifféremment les deux formes successivement prises par l'organisme. 
un instrument de gestion centrale des circonscriptions de sécurité publique, ces chiffres ont peu à peu alimenté la communication politique à mesure que le débat sur «l'insécurité » s'imposait, au détour des années quatre-vingt, au sommet des agendas politiques et médiatiques (Mucchielli, Robert, 2002).

Ce double usage des chiffres, comme instrument de pilotage de l'action policière et comme outil de communication, se développe de façon sensible à partir de l'accession de Nicolas Sarkozy au ministère de l'Intérieur en mai 2002 (Monjardet, 2006). Le nouveau ministre et son équipe s'emploient à instaurer une «culture du résultat» encadrée par des objectifs statistiques, qui modifie les critères d'évaluation de la performance des services. Cette «managérialisation » de la politique policière permet d'alimenter la communication politique du ministère de l'Intérieur (Matelly, Mouhanna, 2007). Sur la base de cette stratégie et au moment de concourir à la magistrature suprême, Nicolas Sarkozy a pu se prévaloir d'un bilan officiel indiquant que les statistiques de la «délinquance générale» avaient baissé d'un peu moins de $10 \%$ depuis 2002, alors qu'elle aurait augmenté d'environ $17 \%$ sous le gouvernement Jospin (Mucchielli, 2008). Les ministres de l'Intérieur successifs ont poursuivi sur la même lancée. Deux chiffres sont particulièrement mis en avant aux niveaux médiatiques, politiques et administratifs 3 : celui de «la délinquance générale», qui totalise l'ensemble des crimes et délits enregistrés sur une année, et permet une appréciation en tendance; le taux d'élucidation général (nombre total de faits élucidés par rapport au nombre total de faits constatés), censé mesurer l'efficacité de l'action des services de police.

\section{Statistiques policières : le succès paradoxal d'une catégorie controversée}

Les dernières années accélèrent donc une histoire de la publicisation des chiffres de la délinquance qui est d'abord celle de son appropriation progressive par le gouvernement, les partis politiques et les médias nationaux. Néanmoins, cet usage croissant des statistiques policières dans le débat public contraste singulièrement avec la faible crédibilité généralement accordée à ces dernières. La critique de ces indicateurs supposés de l'évolution de la délinquance porte à la fois sur la fragilité intrinsèque de l'outil statistique et sur les corrections plus ou moins avouables qu'il peut subir à des fins politiciennes (voir encadré).

3. Le chiffre de la délinquance générale comme le taux d'élucidation globale figuraient jusqu'en 2010 dans le projet annuel de performance en matière de sécurité annexé à la loi de finances. 
Les critiques des statistiques policières (État 4001)

Les chiffres de la délinquance utilisés dans le débat public sont souvent ceux tirés d'une nomenclature statistique conçue par les services du ministère de l'Intérieur. Cet index appelé «État 4001 » est composé de 107 postes correspondant à différents types d'infractions répertoriées par le Code pénal. Sans chercher à être exhaustif sur un point déjà largement étayé par ailleurs (Association Pénombre, 1999; Frenais, Ocqueteau, Varly, 2002; Matelly, Mouhanna, 2007; Mucchielli, 2008; Robert, Zauberman, 2011), on doit rappeler que deux ensembles d'observations sont régulièrement avancés vis-à-vis des enseignements susceptibles d'être retirés de cet indicateur. Premièrement, la construction des principaux agrégats statistiques utilisés dans le débat public est fortement sujette à caution. En particulier, un même agrégat recouvre souvent des évolutions très contrastées. Il en est ainsi notamment du chiffre de la délinquance générale, dont la baisse continue depuis dix ans s'explique essentiellement par la baisse des vols de voitures (globalement non imputable à l'action policière) et masque une augmentation constante de l'enregistrement des atteintes auxpersonnes.

Deuxièmement, les chiffres constitués reflètent moins l'évolution de la délinquance elle-même que les attitudes des victimes et les pratiques des services de police. D'abord, les faits enregistrés dépendent du comportement des victimes, qui décident ou non de déposer plainte. Cet élément est fondamental si l'on considère que l'augmentation continue des atteintes aux personnes observées depuis la fin des années soixante-dix s'explique en grande partie par la plus grande propension des individus à déposer plainte pour un même fait (Mucchielli, 2008). L'évolution statistique des différentes catégories de délit dépend ensuite du classement opéré par le policier (qui peut, par exemple, déclasser une tentative de cambriolage en simple effraction). Enfin, les faits relevés à l'initiative de l'activité des services de police dépendent parfois fortement des orientations du pouvoir politique. En privilégiant - comme ce fut le cas ces dix dernières années - des cibles «faciles» pour lesquelles la constatation d'un fait s'accompagne simultanément de son élucidation (type infractions à la législation sur les titres de séjour), le pouvoir politique s'assure d'obtenir, par le biais des services de police, un «bon » taux général d’élucidation.

Une critique politique vient généralement compléter la discussion des problèmes intrinsèques posés pour l'E 4001, qui souligne que la construction des chiffres de la délinquance comme son commentaire public sont soumis à des tentatives de manipulation. Celles-ci peuvent notamment être suggérées de manière plus ou moins explicite par le pouvoir politique, incitant les fonctionnaires de police à procéder à différents ajustements (refus d'enregistrer une plainte, etc.) susceptibles de modifier les chiffres (Mattelly, Mouhanna, 2007). La création de l'Observatoire national de la délinquance en 2003 a précisément été pensée comme une réponse aux critiques régulières sur le caractère peu transparent de la production des statistiques ministérielles4. Mais les biais relevés n’ont été que

4. La création de l'OND, entérinée sous le premier ministère Sarkozy, fait suite à un rapport parlementaire rédigé à la demande de Lionel Jospin, alors Premier ministre. Voir Christophe Caresche, Robert Pandraud, Rapport de la Mission parlementaire relative à la création d'un observatoire de 
partiellement corrigés. Le «chiffre unique» de la délinquance a notamment été officiellement abandonné par l'ONDRP, les « enquêtes de victimation » réalisées par l'INSEE ont été annexées (Ocqueteau, 2012). Néanmoins, les agrégats proposés par l'Observatoire sont des reconstructions a posteriori qui demeurent toujours adossées aux chiffres collectés par les services du ministère de l'Intérieur.

\section{La sécurité au centre d'une compétition sur enjeux}

En parallèle, il faut souligner que le PS a majoritairement fait le choix, depuis le milieu des années quatre-vingt-dix, de s'emparer du thème de la sécurité, dont ses responsables estiment qu'il est trop coûteux d'un point de vue électoral d'en laisser le monopole à la droite. La sécurité, sous l'impulsion des maires et de certains entrepreneurs de cause, constitue désormais l'une des préoccupations majeures autour desquelles les élus socialistes ont pris l'habitude d'intervenir. L'une des manifestations de l'institutionnalisation des prises de position du PS sur ces questions tient notamment dans la création inédite, en 2000, d'un secrétariat national à la sécurité. Celui-ci a permis au parti à la fois de développer des ressources en termes d'expertise policière et de multiplier les interventions médiatiques sur le sujet (Cos, 2012). Si, dans un premier temps et dans la foulée de l'arrivée de Nicolas Sarkozy Place Beauvau, le «miracle de la sécurité» semble fonctionner - y compris aux yeux de l'opposition (Mouhanna, 2007) -, la campagne de Ségolène Royal en 2007, comme l'avait été celle de Lionel Jospin en 2002, accorde une large place aux questions d'ordre et de sécurité. C'est ce contexte qu'il faut avoir à l'esprit au moment d'analyser les choix rhétoriques du Parti socialiste.

\section{Effet de position, logique d'opposition : l'usage stratégique des chiffres entre délégitimation et instrumentalisation}

Quelles ont été, sous la dernière mandature, les réactions des élus socialistes face aux chiffres divulgués par le gouvernement et la majorité? Cherchent-ils à reprendre ces chiffres? Et si oui, à quelle(s) occasion(s) et comment? On s'est intéressé à la fois aux communiqués de presse diffusés par le parti sur les questions de sécurité, aux différents types d'intervention de ses élus à l'Assemblée nationale et au Sénat5, ainsi qu'aux ouvrages publiés par ses responsables qui

la délinquance, janvier 2002. Le débat de l'époque atteste du fait que, loin d'être confinée à un cercle de spécialistes, cette controverse autour des chiffres a parfois occupé une place importante dans le débat médiatique (Ocqueteau, 2005).

5. Nous avons étudié l'ensemble des communiqués traitant des questions de sécurité depuis leur mise en ligne sur le site internet du parti le 26 octobre 2005, jusqu'au 31 décembre 2011 (n $=65$, à partir de parti-socialiste.fr). Pour les interventions à l'Assemblée Nationale et au Sénat, nous avons procédé à une recherche avec le mot-clé «État 4001 » sous l'actuelle mandature. 
traitent des questions de délinquance. Ces différents supports discursifs ont été soumis à une analyse qualitative adossée à quatre ensembles de variables : les contextes d'énonciation, la place et les formes des polémiques interpartisanes, le degré de sophistication des discours sur les chiffres ainsi que le niveau de distanciation vis-à-vis de l'appareil statistique en matière de délinquance.

\section{La conversion des sources officielles en ressources polémiques}

Quelques opérations sommaires réalisées à partir des communiqués de presse rédigés par le secrétariat national du Parti socialiste révèlent que la dimension chiffrée du débat sur la délinquance occupe une place relativement importante. Entre le 26 octobre 2005 et le 13 septembre 2011, on compte 65 communiqués comportant une évocation d'un ou de plusieurs chiffres de la délinquance, soit un peu moins d'un communiqué par mois en moyenne. Ces 65 textes représentent en outre environ $40 \%$ des 163 communiqués traitant des questions de sécurité relevés sur la période.

Cette place importante occupée par la dimension chiffrée du débat sur l’insécurité procède notamment du fait que si la mensualisation de la publication des statistiques policières, à partir de 2002, a d'abord servi une stratégie ministérielle (Matelly, Mouhanna, 2007), elle a aussi été pleinement exploitée par l'opposition, qui s'est largement focalisée sur un chiffre en particulier : l'augmentation continue des atteintes aux personnes.

Mois après mois, les statistiques de la délinquance rendues publiques par l'Observatoire National confirment la gravité de la montée de la violence dans notre pays. Depuis le début de l'année 2006 , en quatre mois, les violences contre les personnes ont augmenté de plus de $8 \% .^{6}$

Ce phénomène de convergence institutionnelle entre, d'une part, la mensualisation de la publication des chiffres par l'ONDRP, et d'autre part le travail d'analyse produit au niveau du secrétariat national, participe à sa manière de la circulation et la stabilisation de ces grandeurs chiffrées comme figure rhétorique imposée.

De ce point de vue, il faut également souligner que la publication par l'ONDRP, à partir de 2007, des résultats des enquêtes de victimation a multiplié les sources chiffrées disponibles. La conséquence de cette innovation institution-

Si cette entrée ne donne pas accès à l'ensemble des interventions portant sur l'évolution de la délinquance, elle permet de cibler les débats les plus techniques et donne un aperçu des controverses en situation d'interactions. Les documents et interventions trouvés sont issus des sites http://recherche2.assemblee-nationale.fr/ et www.senat.fr. Ce travail intègre également une série d'entretiens avec certains des responsables politiques (députés, sénateurs, secrétaires nationaux, membres élus du conseil d'orientation de l'ONDRP) ou administratifs (haut fonctionnaire policier, chef statisticien de l'ONDRP) les plus impliqués dans ces questions.

6. Communiqué de presse de Delphine Batho, secrétaire nationale PS à la sécurité entre 2003 et 2008,16 mai 2006 . 
nelle tient à ce que l'opposition a pu faire référence à ce qu'il est d'usage d'appeler le «chiffre noir» de la délinquance, soit la somme des crimes et délits objectivement commis chaque année indépendamment de ce qu'enregistrent les services de police. L'invocation du résultat de ces enquêtes a ici une triple utilité argumentative : elle permet de pointer le caractère artificiel des bilans officiels, d'en dénoncer la dimension complaisante et d'établir un contre-diagnostic alarmiste sur la situation :

Le Parti socialiste attire l'attention sur la gravité de la situation que révèle l'enquête nationale de victimation rendue publique aujourd'hui par l'Observatoire National de la Délinquance. En une année, plus de 4 millions de personnes ont été victimes au moins d'une agression, là où les statistiques des faits constatés ne recensent que 400000 atteintes aux personnes. L'écart entre la réalité vécue par nos concitoyens et les chiffres du ministre de l'Intérieurva donc de 1 à 10 . On est bien loin des bulletins de victoire régulièrement brandis par Nicolas SARKOZY et cette enquête discrédite tous les discours du ministre. ${ }^{7}$

Le cas de l'usage des enquêtes de victimation est intéressant en ce qu'il rend compte de la conversion des chiffres disponibles en «cartouches de science » (Lemoine, 2008) mobilisables à des fins polémiques. Les appréciations en tendance délivrées par ce type d'enquêtes ${ }^{8}$ plaident plutôt pour une stabilisation, depuis dix ans, des atteintes aux personnes. Or, lorsque les responsables socialistes mobilisent ces chiffres, ceux-ci ne sont plus destinés à évaluer le bilan de la droite sur la durée, mais à insister sur la réalité brute de cette délinquance à un moment donné. Les responsables socialistes peuvent ainsi espérer marquer l'opinion, attirer son attention sur l'échec présumé du gouvernement, tout en bénéficiant de la légitimité de la source invoquée.

Cette instrumentalisation du caractère officiel des chiffres par la conversion d'une source légitime en ressource polémique s'opère parfois directement, en citant un acteur consensuel du débat public (par exemple les chiffres des atteintes aux personnes, cités dans un rapport de la Cour des comptes abondamment exploité au moment de sa parution)9. Mais les responsables socialistes ont parfois à construire des correspondances entre les chiffres et les caractères officiel et politique de leur provenance. Quand, durant son mandat de secrétaire nationale à la sécurité entre 2003 et 2008, Delphine Batho oppose les chiffres de l'ONDRP, supposé indépendant, aux chiffres avancés par le ministère de l'Intérieur, Jean-Jacques Urvoas, qui occupe les mêmes fonctions depuis 2009, considère quant à lui que les analyses de l'ONDRP sont calquées sur celles du gouvernement.

7. Communiqué de presse de Delphine Batho, 19 décembre 2006.

8. Que ce soit celles réalisées par l'INSEE sous la responsabilité de l'OND intitulées «Cadre de vie et sécurité », ou celles proposées par le CESDIP ou l'IAURIF (Robert, Zauberman, 2011).

9. L'organisation et la gestion des forces de sécurité publique, Rapport public thématique, Cour des comptes, juillet 2011. 
Les premiers résultats de l'enquête de victimation de l'Observatoire National de la Délinquance confirment la prégnance d'une violence généralisée dont plus de 2 millions de personnes ont été victimes en France au cours des deux dernières années [...]. Ces chiffres discréditent les statistiques régulièrement présentées par le ministère de l'intérieur. ${ }^{10}$

Jean-Jacques Urvoas estime ainsi que :

Pour les violences physiques, le rapport du très sarkozyste Observatoire national de la délinquance note, dans une formule qui ferait rire si le sujet n'était pas si grave, une «fin d'accentuation de la hausse». De l'art de présenter une dégradation de la situation sous un angle positif !11

Les usages des chiffres de l'ONDRP donnent ainsi à voir les variations des profits de légitimité qu'on escompte retirer d'une source officielle : une version positive oppose la légitimité scientifique aux visées politiciennes du gouvernement, quand une version négative cherche à retourner la politisation de l'Observatoire contre son supposé chef «officieux» ${ }^{12}$.

\section{Homologies rhétoriques entre droite et gauche}

Une série d'homologies émerge ainsi au sein des stratégies discursives des deux adversaires : la mensualisation des chiffres organisée par N. Sarkozy est exploitée par l'opposition pour nourrir une critique à la fois régulière et «en temps réel» de l'action gouvernementale; à la stratégie d'incarnation de la lutte contre la sécurité portée par N. Sarkozy répond la stratégie d’imputation organisée par le PS ; à la reprise régulière du «chiffre unique » de la délinquance répond le pourcentage «accablant» des violences aux personnes.

De fait, l'usage des chiffres de la part de l'opposition socialiste ne s'apparente que de façon lointaine à un exercice d'objectivation de la réalité délinquante. Le rapport aux chiffres s'insère fondamentalement dans la trame des échanges polémiques au sein du champ politique. L'analyse que propose le discours politique d'un problème donné est en effet sans cesse tributaire de la volonté d'administrer des coups et d'imposer des coûts à l'adversaire (Le Bart, 1998). Le propos de Jean-Jacques Urvoas synthétise ici l'analyse de la plupart des élus rencontrés : "Il y a une part de mauvaise foi qui est consubstantielle au débat politique, oui bien sûr, oui c'est normal. Parce que je sais que pour frapper l'opinion il faut un chiffre fort. $»^{13}$

10. Communiqué de presse de Delphine Batho, 12 novembre 2007.

11. Communiqué de presse de Jean-Jacques Urvoas, secrétaire national PS à la sécurité depuis mai 2009, 12 novembre 2009.

12. Entre juillet 2004 et octobre 2009, l'OND dépendait directement du ministère de l'Intérieur. Depuis cette date, l'ONDRP dépend du Premier ministre.

13. Entretien avec Jean-Jacques Urvoas, 15 décembre 2011, Assemblée nationale, Paris. 
Au-delà des spécificités liées à la dimension chiffrée de la controverse, c'est au fond l'économie générale des débats interpartisans qui est au principe de ce rapport opportuniste aux sources de savoir disponibles.

Néanmoins, souligner l'influence que peut avoir la position occupée par les responsables politiques sur leurs prises de position ne revient pas à conclure que les pratiques des agents sont entièrement réductibles à un calcul cynique et rationnel entre coûts et intérêts. Les effets de position se combinent en permanence avec les représentations véhiculées par les agents investis dans les luttes politiques.

\section{La force d'un sens politique commun en matière de sécurité}

À ce stade, une question basique mais décisive doit être soulevée : les politiques sont-ils informés des distorsions massives qui biaisent les diagnostics chiffrés de la délinquance? La réponse n'est évidemment pas univoque : elle dépend à la fois des agents considérés et du niveau de technicité du débat. Mais elle conduit à faire ressortir les croyances dominantes portées par les responsables politiques.

\section{Une maîtrise superficielle de l'enjeu des chiffres}

Outre les contraintes - notamment temporelles - qui s'imposent aux interventions des élus en séance et les effets de la division du travail interne aux groupes parlementaires, il faut souligner le caractère coproduit des interventions publiques des hommes politiques. Les rapports parlementaires, par exemple, sont souvent coécrits avec l'appui de collaborateurs de groupes, et même parfois avec le soutien d'un personnel administratif souvent bien informé des problèmes posés par la statistique policière. Si beaucoup de parlementaires ont également un mandat de maire qui peut les mettre régulièrement aux prises avec les chiffres policiers au niveau local (Mouhanna, 2005), cette connaissance paraît souvent très rudimentaire. Il est ainsi frappant de constater la faible appropriation des enjeux relatifs aux chiffres, y compris chez les agents les plus régulièrement aux prises avec ces questions. Le témoignage suivant, qui émane d'un sénateur socialiste dont on peut présumer que sa présence au sein du conseil d'orientation de l'ONDRP lui a permis d'approcher les dysfonctionnements de la statistique policière, est significatif :

[J'utilise] des chiffres très globalisés. Très globalisés. Dès qu'on rentre dans les sous, sous-analyses de ceci ou de cela euh... j’y échappe... j’y échappe. La tendance générale suffit. [...] Mais les chiffres d'analyse de, de masse, sont intéressants. Et leur évolution, surtout. En fait c'est ça qui compte. C'est comme la température du 
malade! Si un mec a tout le temps 39, on peut finir par croire que son biotope est à 39. C'est pas forcément grave. Mais un mec qui passe de 37 à 42, il en a plus pour longtemps. ${ }^{14}$

Tout se passe en effet comme si, pour les responsables politiques ayant à se prononcer régulièrement sur ces questions, les interventions s'appuyaient généralement sur une forme de sens commun bricolé, attestant d'une méfiance générale vis-à-vis des chiffres en même temps que d'un attachement à la force supposée des statistiques sur le long terme. Le propos de François Rebsamen, chargé des questions de sécurité dans le cadre de la campagne présidentielle de François Hollande, concentre ici la croyance des élus dans la crédibilité de certains chiffres :

Je n'imagine pas qu'il n'y ait pas un outil comme l’État 4001. Il reste pertinent à condition de ne pas lui faire dire ce qu'il ne peut pas dire. Ainsi, les chiffres sur les violences aux personnes constituent un élément fiable. Mais il ne faut pas additionner les choux et les carottes. Et il faut s'intéresser aux tendances à la hausse ou à la baisse sur de longues périodes. Ainsi, la diminution historique des assassinats depuis trente ans est incontestable. De même que la hausse des violences aux personnes qui a progressé sous Lionel Jospin et a pratiquement doublé depuis que la droite est au pouvoir. ${ }^{15}$

\section{Croyance dans les chiffres et sentiment politique d'insécurité}

Cette foi dans la fiabilité des statistiques policières sur le long terme - en particulier en matière d'atteintes aux personnes - acquiert d'autant plus un caractère d'évidence qu'elle répercute et s'encastre dans une croyance policière solidement ancrée. Si le chiffre de la délinquance générale fait depuis quelque temps l'objet de vives contestations ${ }^{16}$, il demeure encore une référence incontournable pour de nombreux agents administratifs et policiers - et ce d'autant que les syndicats y ont jusqu'à récemment trouvé un certain intérêt (Matelly, Mouhanna, 2007).

L'hypothèse d'une judiciarisation des faits sociaux, qui rendrait compte d'une part importante de l'augmentation des violences interpersonnelles enregistrées, semble également trouver peu d’échos au sein des services ministériels. Si ce constat renvoie au fait que cette interprétation est relativement récente, y compris au sein des sciences sociales, il trouve plus fondamentalement ses racines dans la relative étanchéité qui sépare aujourd’hui savoirs aca-

14. Entretien avec Charles Gautier, sénateur-maire de Saint-Herblain, président du Forum Français pour la Sécurité Urbaine, membre du Conseil d'orientation de l'ONDRP, 29 décembre 2011, mairie de Saint-Herblain.

15. "Présidentielle 2012 : François Rebsamen présente à AISG son équipe et ses premières pistes de travail sur la sécurité », dépêche AISG nº 4915, 13 décembre 2011.

16. «État 4001 : Alain Bauer demande “d'abandonner l'outil statistique devenu archaïque, obsolète et anti-fonctionnel” », dépêche AISG n² 2298, 21 janvier 2011. 
démiques et savoirs d'État depuis la reprise en main de l'Institut des Hautes études sur la sécurité intérieure (IHESI) ${ }^{17}$ par des hauts fonctionnaires policiers proches du pouvoir en place (Monjardet, Ocqueteau, 2005). C'est ainsi la relative marginalisation des institutions (le CESDIP notamment) porteuses d'analyses et de cadrages alternatifs qui tend à renforcer le caractère d'évidence que revêt aux yeux des agents l'augmentation statistique des violences aux personnes.

L'usage de ces chiffres, et la propension à accorder plus ou moins de crédit à tel ou tel d'entre eux, est également tributaire des discours médiatiques autant que des représentations générales de la délinquance par les agents qui les mobilisent. Ces représentations agrègent des jugements, déductions, analyses souvent tirées de l'expérience ordinaire; les chiffres viennent alors confirmer et valider « objectivement» des représentations qui leur préexistent.

Question : L'augmentation des atteintes aux personnes, qui pour le coup est enregistrée depuis pas mal de temps, ça vous l'interprétez comment? - Réponse : [silence] Par la maladie de la société, qui est quand même une société de violence ... Cette société de violence, donc là elle se manifeste par des agressions, mais on la sent aussi - enfin quand on est dans le terrain etc., y compris dans le... dans le parlé, dans le parlé. Euh, le ton, le vocabulaire, etc., dans les réunions publiques, ou dans les contacts directs, sont plus âpres que ce qu'on a pu connaître. Enfin ça monte quoi, ça monte ! [...] Alors depuis combien de temps je sais pas le dater, mais, mais, mais... elle est démon... enfin tout le monde en témoigne, en tous les cas. ${ }^{18}$

Ce tropisme sécuritaire fondé sur une croyance forte dans l'augmentation de la violence, aujourd'hui très prégnant y compris parmi les élus de gauche, constitue le terreau dans lequel s'enracine la reprise routinière de chiffres alarmistes. Ces différents éléments convergent alors vers la production d'une forme de sens politique commun en matière de chiffres de la délinquance qui peut être schématiquement résumé comme suit : les statistiques policières sont imparfaites; mais l'usage d'un même outil à travers le temps permet une appréciation en tendance de la délinquance ; la société est de plus en plus violente; le chiffre des atteintes aux personnes en est un bon indicateur ${ }^{19}$.

\section{La variabilité des incitations à « jouer le jeu »}

L'expression de ce bricolage semi-savant peut toutefois prendre des formes plus ou moins sophistiquées selon les contextes d'énonciation : le métier

17. Depuis 2010, Institut national des hautes études de la sécurité et de la justice (INHESJ).

18. Entretien avec Charles Gautier.

19. La première note de bas de page de l'ouvrage de Manuel Valls, introduite après le mot « chiffre », indique que «si l'outil statistique est souvent instrumentalisé et corrigé pour donner des résultats cosmétiques meilleurs - j'y reviendrai longuement dans le chapitre II -, il ne parvient pas, sur la longue tendance, à cacher grand-chose». M. Valls, 2011, p. 9. 
politique n'est pas uniforme et se décline différemment selon les scènes d'expression dans lesquelles interviennent les élus. La sociologie des rôles politiques enseigne en outre que ceux-ci sont modelés par les dispositions initiales des agents qui les endossent (Lagroye, 1997).

\section{Une division du travail politique fonction des scènes locales et nationales}

Comme les contextes d'énonciation, les supports argumentatifs mobilisés sont plus ou moins propices à une critique de la donne statistique. Cette distribution est particulièrement notable lorsqu'elle rencontre les logiques de multipositionnalité dans lesquelles sont pris les élus. Ainsi en est-il de l'articulation local/ national qui recouvre, pour une large part, la division individuelle du travail politique entre gestionnaire et orateur. Car si les hommes politiques font un usage abondant des chiffres policiers lorsqu'ils sont en représentation à l'Assemblée ou au Sénat, ces mêmes grandeurs ne constituent que très marginalement des éléments d'orientation pour l'action publique mise en œuvre à l'échelon local.

Quand on est dans une période où le débat politique est tendu, les chiffres représentent quelque chose parce qu'ils sont un terrain d'affrontement [...]. La réalité localement c'est que euh... ils ont que peu d'importance. [...] C'est quelque chose de très complexe, moi les chiffres je... comme tout le monde, c'est comme celui qui vous parle des sondages et qui dit que ça n'a pas d'importance pour lui. Les chiffres on les attend, on essaie de les avoir. Quand les préfets ne nous les envoient pas on les lui réclame, parce que ça permet d'établir des comparaisons avec d'autres villes. Ça peut permettre de regarder par rapport à un dispositif que l'on a mis en place s'il a eu un intérêt par rapport à... bon. Et puis, pour autant, faut pas chercher quand même à leur donner plus d'importance que ça. ${ }^{20}$

\section{Les dispositions réformatrices au révélateur des controverses autour de l'ONDRP}

L'ONDRP constitue de ce point de vue une scène particulièrement intéressante où sont mises en tension les positions respectives de parlementaires également investis d'un mandat local. En tant qu'espace formellement pluraliste de production des savoirs d'État, l'ONDRP met en outre directement aux prises les élus avec la fabrique des agrégats statistiques. On peut donc se demander si cette scène institutionnelle constitue une « situation d'espace public » (Desrosières, 1992) au sein de laquelle les politiques chercheraient à mettre en discussion les fondations du débat sur les chiffres de la délinquance.

20. Entretien avec Bruno Le Roux, député socialiste de Seine-Saint-Denis, actuel président du groupe socialiste à l'Assemblée nationale, ancien maire d’Épinay-sur-Seine et délégué national du PS aux questions de sécurité et de police entre 1995 et 1997. 
De fait, l'ensemble des acteurs rencontrés ayant siégé ces dernières années au Conseil d'orientation (COR) témoignent de la passivité des responsables politiques en séance ${ }^{21}$. Ce ressenti est confirmé tant par les comptes rendus disponibles sur le site que par les rapports annuels rendus depuis 2005 et dans lesquels sont censées figurer les remarques, observations ou critiques des membres du COR : seul Jacques Mahéas, sénateur socialiste, maire de Neuillysur-Marne et membre entre 2003 et 2010 , est intervenu à deux reprises ${ }^{22}$. Les quatre responsables socialistes que nous avons interrogés sur les tenues du COR insistent pour souligner combien les inégalités de ressources intellectuelles biaisent la nature des échanges entre élus et responsables administratifs. Pour l'essentiel, les utilisations des travaux de l'ONDRP restent prises dans l'ordinaire des controverses politiques, dès lors qu'elles peuvent opportunément servir une critique du bilan gouvernemental. L'investissement par les politiques à l'Observatoire catalyse au fond le rapport à la fois stratégique et distant entretenu par ces politiques vis-à-vis du débat sur les chiffres 23 .

Par ailleurs, les prises de position des socialistes ayant le plus voix au chapitre ces dernières années sur les questions de sécurité donnent à voir deux types de logiques assez contradictoires qui régissent une partie du rapport des agents aux savoirs académiques et/ou d'État. Le rapport au chiffre peut d'abord fonctionner comme le révélateur des effets de connivence qui influent parfois sur l'économie polémique du champ politique. Le rapport aux chiffres est inséparablement un rapport aux institutions qui les produisent, et parfois plus immédiatement encore aux hommes qui occupent ces institutions. Le cas de l'ancien député-maire socialiste d'Évry, "spécialiste» des questions de sécurité et désormais ministre de l'Intérieur Manuel Valls est révélateur en ce sens. Son ouvrage sur la sécurité, paru en 2011, reprend abondamment les chiffres de l'ONDRP sans jamais chercher à les discuter ${ }^{24}$. L'une des raisons de cette propension à reprendre tels quels les chiffres d'un organisme réputé proche du pouvoir - organisme dont l'ouvrage ne discute jamais des ambiguïtés statutaires -, tient aux relations entretenues par l'auteur avec le président d'alors du conseil d'orientation de l'ONDRP, Alain Bauer. Ce dernier, outre son

21. Élus par ailleurs faiblement assidus aux réunions du COR. Pour l'année 2011 - seule année pour laquelle les PV de présence sont disponibles sur le site de l'INHESJ -, sur les quatre réunions et pour les six élus nommés, on comptabilise un taux de présence de $37,5 \%$, qui tombe à $25 \%$ si l'on exclut les membres de l'ancienne majorité.

22. Voir «Remarques de Jacques Mahéas » dans les rapports annuels de l'OND-RP en 2008 et 2009.

23. D’après le récit que propose Frédéric Ocqueteau, directeur de recherche au CNRS, spécialiste des questions de sécurité et membre du COR de l'ONDRP entre 2004 et 2009, une large part des interventions en séance des personnalités politiques nommées nationalement consistait à réclamer l'installation d'observatoires locaux de la délinquance dans leurs communes respectives. Nous remercions l'auteur de nous avoir transmis le document de travail faisant état de ce récit. Voir également Ocqueteau, 2005, 2012.

24. Voir sur ce point la critique de Laurent Mucchielli, «La posture autoritaire et populiste de Manuel Valls», sur insecurite.blog.lemonde.fr, 5 juin 2011. 
passage par l'UNEF-ID et ses attaches historiques parmi les anciens réseaux rocardiens, est aussi tenu par des liens affectifs avec certains membres de l'opposition. Il est un ami de jeunesse et le parrain du deuxième fils de Manuel Valls et, d'après plusieurs témoignages, conseille encore activement ce dernier sur ses positions en matière de sécurité. Ce type de relations participe donc, à sa manière, du consensus plus ou moins stable qui domine au sein du champ sur la légitimité de l'ONDRP et de son exploitation des données policières.

Cette stratégie d'évitement de la controverse autour de l'ONDRP, de son statut comme de ses travaux, est intéressante à mettre en miroir avec celle, inverse, de Jean-Jacques Urvoas. Si ce parallèle ne peut être ici qu'esquissé, il atteste de la variation relative des rapports politiques aux instruments de connaissance. Jean-Jacques Urvoas, secrétaire national à la sécurité, également membre du COR de l'ONDRP, a en effet transposé dans le champ politique un habitus universitaire importé de ses fonctions de maître de conférences en droit public. Au départ totalement étranger aux problématiques de sécurité, ce député alors investi de son premier mandat parlementaire s'est largement engagé depuis sa prise de fonction dans la production de notes, rapports, articles et ouvrages sur les questions de police et de sécurité. Il en résulte une sollicitation régulière de certains chercheurs en sciences sociales (notamment du CESDIP) et une approche technicienne assez poussée des différents sujets dont il a à traiter. Dans un ouvrage paru en 2011, il met en avant différentes pistes de réforme d'un Observatoire dont il rappelle la forte coloration politique. Constatant que «la nasse s'est resserrée sur le contrôle tentaculaire de la connaissance » en matière de délinquance, il conclut : «Supposé rationaliser, consolider et dépassionner l'évaluation et l'analyse de la délinquance, [l'ONDRP] est devenu un instrument de crédibilisation des chiffres policiers et des stratégies du ministère de l'Intérieur. » 25 De ce constat, il retire la nécessité de supprimer l'Observatoire et de charger l'INSEE de la production des statistiques criminelles, cherchant ainsi à corriger les défauts d'un indicateur statistique jugé trop politique. S’il ne faut pas négliger le fait que ces velléités réformatrices s’insèrent dans une stratégie individuelle et distinctive, cet exemple suggère qu'à certaines conditions, les dispositions professionnelles acquises antérieurement par certains agents en dehors du champ politique peuvent conduire à une forme de contestation de la configuration institutionnelle et cognitive dominante ${ }^{26}$.

25. Urvoas, 2011, p.55-60. Le texte de présentation du programme du parti socialiste en matière de sécurité, largement rédigé par J.-J. Urvoas et rendu public en octobre 2010, reprend cette proposition dans son dernier point. Voir Parti socialiste, Pacte national de protection et de sécurité publique, Créteil, octobre 2010. Elle a toutefois été implicitement abandonnée par François Rebsamen, référent «sécurité » de la campagne présidentielle de François Hollande. «Présidentielle 2012 : François Rebsamen présente à AISG son équipe et ses premières pistes de travail sur la sécurité», dépêche AISG nº 4915, 13 décembre 2011.

26. Sur les relations entre dispositions sociales et rapport aux chiffres, voir Pénissat, 2007. 
Pour conclure, on doit d'abord souligner les homologies qui ont relié les usages des chiffres par l'opposition socialiste à ceux de l'ancienne majorité au pouvoir : le caractère régulier de la critique comme la focalisation de celle-ci sur certains chiffres particuliers opère en miroir de la stratégie développée par les adversaires du PS. Pour rendre compte de cette homologie, il faut reconstituer la configuration discursive qui met en relation les caractéristiques intrinsèques des chiffres mobilisés (fortement critiqués mais suffisamment pluriels pour faire l'objet d'appropriations concurrentes) et la position du Parti socialiste dans l'espace politique. Cette position n'est pas réductible au statut d'opposant, mais intègre également la stratégie de moyen terme du PS de débattre avec la droite "sur son terrain », et les dispositions respectives des agents impliqués dans les controverses. Les éléments discursifs analysés résultent ainsi de la confrontation de la logique stratégique et des croyances de sens commun, des scènes d'expression politique et des dispositions socioprofessionnelles qui structurent les prises de positions des professionnels de la politique. Il reste à présent à considérer dans quelle mesure ce faisceau de relations se trouve modulé par la pratique gouvernementale.

\section{Références}

Association Pénombre, 1999, Chiffres en folie. Petit abécédaire de l'usage des nombres dans le débat public et les médias, Paris, La Documentation française.

Aubusson de Cavarlay Bruno, 1996, "Les statistiques de police. Méthodes de production et conditions d'interprétation », Mathématiques, Informatique, Sciences Humaines, $\mathrm{n}^{0} 134$.

BeLorgey Nicolas, 2010, L'hôpital sous pression. Enquête sur le «nouveau management public », Paris, La Découverte (Textes à l'appui - Enquêtes de terrain).

BezÈs Philippe, 2009, Réinventer l'État. Les réformes de l'administration française (1962-2008), Paris, PUF (Le lien social).

BRUno Isabelle, 2008, À vos marques, prêts... cherchez! La stratégie européenne de Lisbonne, vers un marché de la recherche, Paris, Le Croquant (Savoir/Agir).

Cos Rafaël (à paraître), «Le parti socialiste et la régulation politique des désordres. L'émergence d'un nouvel entrepreneur de sécurité », Regards croisés sur la régulation sociale des désordres, E. Boucher éd., Paris, L'Harmattan (Logiques sociales).

DESROSIÈrES Alain, 1992, «Discuter l'indiscutable. Raison statistique et espace public», Pouvoir et légitimité. Figures de l'espace public, Paris, Raisons pratiques, n³3.

Frenais Jacques, Ocqueteau Frédéric, Varly Pierre, 2002, Ordonner le désordre. Une contribution au débat sur les indicateurs du crime, Paris, La Documentation française / IHESI.

LAG ROYE Jacques, 1997, « On ne subit pas son rôle », Politix, vol. X, n³ 38. 
LE BART Christian, 1998, Le discours politique, Paris, PUF (Que sais-je?).

LEMOINE Benjamin, 2008a, «Entre fatalisme et héroïsme. La décision politique face au "problème" de la dette publique (2003-2007) », Politix, n 82.

- 2008b, "Chiffrer les programmes politiques lors de la campagne présidentielle 2007. Heurs et malheurs d'un instrument», Revue française de science politique, vol. LVIII.

Matelly Jean-Hugues, Mouhanna Christian, 2007, Police. Des chiffres et des doutes, Paris, Michalon.

MONJARDET Dominique, 2006, «Comment apprécier une politique policière ? Le premier ministère Sarkozy (7 mai 2002-30 mars 2004) », Sociologie du travail, vol. XLVIII.

MONJARDET Dominique, OCQUETEAU Frédéric, 2005, «Insupportable et indispensable, la recherche au ministère de l'Intérieur ", L'État à l'épreuve des sciences sociales, P. Bezes et al., Paris, La Découverte, p. 229-247.

MounannA Christian, 2005, "Coproduction, cohérence ou concurrence? Réflexions sur la coopération élus-policiers en matière de sécurité», Peurs sur les villes, J. Ferret, C. Mouhanna éd., Paris, PUF (Sciences sociales et sociétés).

— 2007, "Le Miracle de la sécurité, vu de l'Intérieur», Mouvements, n¹1-12.

MucCHIELLI Laurent, 2008, "Le "nouveau management de la sécurité" à l'épreuve. Délinquance et activité policière sous le ministère Sarkozy (2002-2007)», Champ pénal, vol.V.

- 2010, "Les techniques et les enjeux de la mesure de la délinquance », Savoirs/Agir, n०93.

Mucchielli Laurent, Robert Philippe, 2002, Crime et sécurité. L'état des savoirs, Paris, La Découverte.

OCQUeTEAu Frédéric, 2005, "Observer les délinquances. Où, comment et pourquoi ? Sur la genèse de l’Observatoire national de la délinquance», Peurs sur les villes, J. Ferret, C. Mouhanna éd., Paris, PUF (Sciences sociales et sociétés).

- 2012 (à paraître), «Une machine à retraiter les outils de mesure du crime et de l'insécurité, l’Observatoire national de la délinquance », Droit et société, vol. I.

PÉNISSAT Étienne, 2007, «Entre science, administration et politique. Produire des statistiques au sein d'un ministère », Socio-Logos, nº 2 .

Robert Philippe, Zauberman Renée, 2011, Mesurer la délinquance, Paris, Presses de Sciences Po.

VALLS Manuel, 2011, Sécurité. La gauche peut tout changer, Paris, Éditions du Moment. UnVoAs Jean-Jacques, 2011, 11 propositions chocs pour rétablir la sécurité, Paris, Fayard / Fondation Jean-Jaurès. 